\title{
The miRNA expression profile of the uveal melanoma
}

\author{
YANG ChengHsun ${ }^{1,2} \&$ WEI WenBin ${ }^{1,2 *}$ \\ ${ }^{1}$ Beijing Tongren Eye Center, Beijing Tongren Hospital, Beijing 100730, China; \\ ${ }^{2}$ Capital Medical University, Ophthalmology \& Visual Science Key Laboratory, Beijing 100730, China
}

Received March 20, 2010; accepted May 20, 2010; published online March 14, 2011

\begin{abstract}
The miRNA expression profile was initially established to investigate its corresponding function in human uveal melanoma. The miRNA expression profile in human uveal melanoma was analyzed by a micro chip technique. The hsa-miRNA expression between four uveal melanomas and four normal uveal tissues was compared. Based on the bioinformatic approach, chip data was analyzed to select out differentially expressed candidate hsa-miRNAs. Real-time quantitative PCR (RT-PCR) was used to confirm the candidate hsa-miRNAs expression in all samples. The results of miRNA microarray chips that matched with RT-PCR were considered as the miRNA expression which was significantly different between normal tissue and uveal melanomas. In four uveal melanomas, expressions of miRNA-20a, miRNA-106a, miRNA-17, miRNA-21, and miRNA-34a were significantly up-regulated, while miRNA-145 and miRNA-204 expression were significantly down-regulated. We used miRNA microarray analysis as a fast, efficient technology to study biological information. The differentially expressed miRNAs may be involved in uveal melanoma pathogenesis, and may help promote the diagnosis and treatment for uveal melanoma.
\end{abstract}

uveal melanoma, miRNA, microRNA microarray, target gene, tumorigenesis

Citation: Yang C H, Wei W B. The miRNA expression profile of the uveal melanoma. Sci China Life Sci, 2011, 54: 351-358, doi: 10.1007/s11427-011-4149-y

Uveal melanoma is the most common primary intraocular malignant tumor in adults, accounting for $5 \%-6 \%$ of all cases of primary systemic melanoma [1]. The onset age, pathological classification, tumor size, infiltration depth to sclera, onset location, and nuclear division of tumor cells are all related to its prognosis. Tumor cells are mainly derived from uveal melanocytes, with characteristics of high malignancy and easy metastasis. The primary cause of death is hepatic metastasis. Once diagnosed with tumor metastasis, the prognosis is extremely poor, where most patients will die within months, with a typical survival period of 2-7 months. Only $13 \%$ of patients survive more than one year. Clinical treatment for this disease includes photocoagulation, radiotherapy, local tumor incision, and eye removal, even though they do not significantly improve the survival rate. Because its etiology and pathogenic mechanism remain

*Corresponding author (email: drwbwei@gmail.com) unknown, it is not yet possible to improve the early diagnosis for uveal melanoma and its metastasis.

Non-coding small RNAs define many eukaryotic cell functions and the impacts of a variety of actions, e.g., gene expression, cell cycle and cellular development [2]. Small RNA primarily comprises short interfering RNA (siRNA) and microRNA (miRNA) [3]. miRNA provides a pathway for uveal melanoma pathogenesis. It carries a phosphoric acid group at the $5^{\prime}$-end and a hydroxyl group at the 3 '-end. It contains 22 nucleotides, which belong to a family of non-encoding regulatory RNA. More than $1 / 3$ of human genes are controlled by miRNA. Because abnormal cellular differentiation is usually the key to tumor formation and miRNA is able to cause abnormal differentiation, the expression of miRNA indicates the status of malignant transformation. Therefore, the miRNA gene is a key factor in human tumors. This study utilized the genome chip technique to screen out miRNA genes in uveal melanoma and to 
explore such specific genes.

\section{Materials and methods}

\subsection{Patient recruitments}

After intraocular local tumor incision, four histologically confirmed uveal melanomas (among them were three cases of uveal melanoma of the spindle cell type and one case of mixed type uveal melanoma with a primarily spindle cell type) at Beijing Tongren Hospital were included in this study. Another four normal donated choroidal samples were also included. Informed consent was obtained from all patients.

\subsection{Sample preparation}

Each sample was equally divided into two sections. One block was stained with standard HE and immunohistochemical methods to confirm tissue characteristics, and the other was stored in a liquid nitrogen refrigerator $\left(-80^{\circ} \mathrm{C}\right)$ for miRNA extraction.

\subsection{Experimental procedures}

(i) The actual procedures for HE staining and immunohistochemical staining are not described here. Tissue samples of confirmed choroidal melanoma were selected. (ii) Total RNA was extracted from tissue blocks or cells, using the single-stepped Trizol method. By the precipitation method with isopropyl alcohol, RNA was condensed. Using a spectrometer for quantification, denatured gel electrophoresis with formaldehyde was employed to test the quality of all RNA (Table 1). RNA, which were qualified by an electrophoretic test, were purified and labeled. (iii) The miRNAs were isolated, by processing $40.0 \mu \mathrm{g}$ of total RNA with the PEG method. The remaining total RNA was stored in $\mathrm{DEPC}$ water in a refrigerator at a temperature of $-80^{\circ} \mathrm{C}$, for further verification by RT-PCR. (iv) Bovine alkaline phosphorase was used for de-phosphorylation and fluorescent labeling of miRNA samples. Using T4 RNA linkase,
CU-cy3 and CU-cy5 were respectively labeled, before the purified and labeled products were isolated. (v) Hybridization and cleansing involved the dissolving of RNA in $20 \mu \mathrm{L}$ of hybridizing solution ( $15 \%$ formamide, $0.2 \% \mathrm{SDS}, 3 \times \mathrm{SSC}$, $5 \times$ Denhardt's) and leaving them at $42^{\circ} \mathrm{C}$ for overnight hybridization. After completing the hybridization, at $42^{\circ} \mathrm{C}$, they were washed in solution of $0.2 \%$ SDS and $2 \times \mathrm{SSC}$ for 4 min, before another 4 min of being washed at room temperature in a $0.2 \times \mathrm{SSC}$ solution. (vi) Tumor and normal tissue samples were randomly paired. (vii) A chip scan was conducted by using a dual-channeled laser scanner of LuxScan 10K/A (CapitalBio Company) after the glass slides were dried.

\subsection{Chip images and data analysis}

(i) Chip data was extracted using LuxScan 3.0 (CapitalBio Company) image analysis software and the images were analyzed, with the signals converted into digital forms. (ii) Data analysis: Each chipset was adjusted and standardized. Based on signal strength and image quality, miRNAs were labeled. Disqualified data was eliminated. The data was analyzed by using the significance analysis of microarrays (SAM), where FDR was controlled within 5\%. Genes of twice the variant expression were screened out and the data was accordingly categorized.

\subsection{RT-PCR}

(i) Primers were designed and synthesized. For testing miRNA, a stemmed, ring-form reverse transcription primer and corresponding PCR primer were designed. (ii) Reverse transcription of cDNA: $0.05 \mu \mathrm{g}$ total RNA, which was stored in $-80^{\circ} \mathrm{C}$ DEPC water, was acquired and reversely transcribed into 1 st cDNA by reverse transcription. The process of reverse transcription involved the following: 30 min at $16^{\circ} \mathrm{C}, 30 \mathrm{~min}$ at $37^{\circ} \mathrm{C}, 10 \mathrm{~min}$ at $70^{\circ} \mathrm{C}$, and a continuous process at $4^{\circ} \mathrm{C}$. (iii) RT-PCR: The $1 \mathrm{st}-\mathrm{cDNA}$, as a template, was used to optimize the PCR system and to confirm primer conditions. With $1^{\text {st }}$-cDNA as a template and an optimized experimental system, the real-time PCR amplifi-

Table 1 miRNA chip analysis

\begin{tabular}{|c|c|c|c|c|c|c|c|}
\hline No. & State & $A_{260}$ & $A_{260 / 280}$ & $A_{260 / 230}$ & Concentration $\left(\mu \mathrm{g} \mu \mathrm{L}^{-1}\right)$ & Total $(\mu \mathrm{g})$ & Results \\
\hline $\mathrm{N} 2$ & Tissue in dry ice & 39.174 & 1.65 & 1.06 & 1.567 & 235.05 & RNA available \\
\hline $\mathrm{T} 1$ & Tissue in dry ice & 95.332 & 1.78 & 1.45 & 3.81 & 762.66 & RNA available \\
\hline $\mathrm{T} 2$ & Tissue in dry ice & 71.814 & 1.80 & 1.28 & 2.87 & 574.51 & RNA available \\
\hline N1 & Tissue in dry ice & 33.354 & 1.78 & 1.25 & 1.33 & 266.83 & RNA available \\
\hline N3 & Tissue in dry ice & 20.805 & 1.64 & 0.99 & 0.83 & 166.44 & RNA available \\
\hline $\mathrm{T} 3$ & Tissue in dry ice & 79.01 & 1.79 & 1.38 & 3.95 & 592.5 & RNA available \\
\hline $\mathrm{T} 4$ & Tissue in dry ice & 36.48 & 1.83 & 1.64 & 1.82 & 182 & RNA available \\
\hline
\end{tabular}


cation commenced. 1.6 $\mu \mathrm{L}$ of $\mathrm{MgCl}_{2}$ was used and the primer concentration was set at $10 \mu \mathrm{mol} \mathrm{L} \mathrm{L}^{-1}$. The annealing temperature was set at $60^{\circ} \mathrm{C}$. It was followed by $10 \mathrm{~min}$ of activation by kinase at $95^{\circ} \mathrm{C}$ and $15 \mathrm{~s}$ of denaturation at $95^{\circ} \mathrm{C}$. The annealing process was performed at $60^{\circ} \mathrm{C}$ and followed by $30 \mathrm{~s}$ of elongation. At $74^{\circ} \mathrm{C}$ and under fluorescence, it was tested for $3 \mathrm{~s}$. This entire process was repeated 40 times. The dissolution curve between $75^{\circ} \mathrm{C}$ and $95^{\circ} \mathrm{C}$ was plotted. (iv) The electrophoresis was performed to test the amplification status of PCR. (v) Data analysis was performed to conclude.

\section{Results}

\section{1 miRNA chip analysis}

\subsubsection{Electrophoresis}

Electrophoretic bands of RNAs are clearly shown in Figure 1 . The ratio of band intensity $28 \mathrm{~S} / 18 \mathrm{~S}$ rRNA was close to $2: 1$, which is considered acceptable for a chip scan.

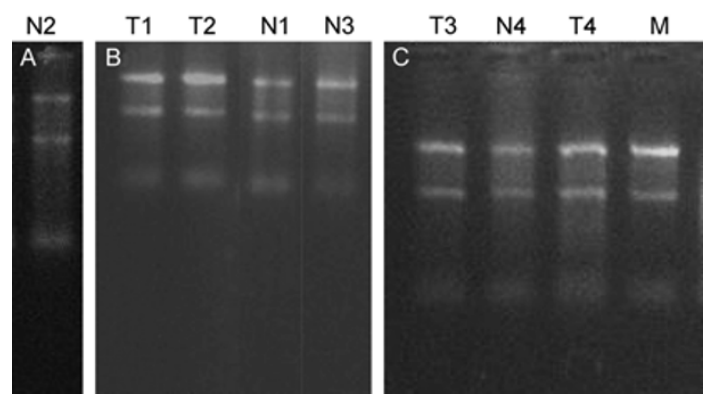

Figure 1 Electrophoretic pattern of total RNA. A, Second channel, N2. B, First channel, T1; second channel, T2; fifth channel, N1; seventh channel, N3. C, First channel, T3; second channel, N4; third channel, T4; M channel, RNA of Hela cells.

\subsubsection{Chip scan}

Pseudo-color map for chip hybridization in Figure 2: cy5 displayed in red color and cy 3 in green color. For a specific signal, the image would be in a sharp green color if a stronger cy 3 signal was present. By contrast, a cy5 signal will display a sharp red color. If the intensities of the two colors were similar, a yellow color would result.

\subsubsection{Screening of miRNA up to a two-fold expression}

The following data included the ratio of the standardized signals of cy 3 and cy 5 in the chip. Score $(d)$ represents the statistical quantity $=$ Numerator $(r) /$ Denominator $(s+s 0)$. Fold Change was the duplication factor of difference. The $Q$-value (\%) was the false discovery rate (FDR) of miRNA being determined as differential miRNA, which was similar to the $P$-value. When the $Q$-value was smaller, the difference was more significant.

Finally, the miRNA chip scan results show that hsamiRNA-20a, 106a, 17, and 34a all had up-regulation in the differential expression, while the hsa-miRNA-145 and 204 had a down-regulated differential expression. These results were verified by RT-PCR (Table 2).

\subsection{RT-PCR}

\subsubsection{Electrophoresis}

The following channels used 1st-cDNAs of samples of N4, N1, N3, N2, T3, T1, T3, and T4 as templates for each electrophoresis result of miRNA. Electrophoresis results show that miRNA had a strong specific response to RT-PCR in Figure 3 .

\subsubsection{RT-PCR results}

In the following Table 2, the expression of hsa-miRNA-
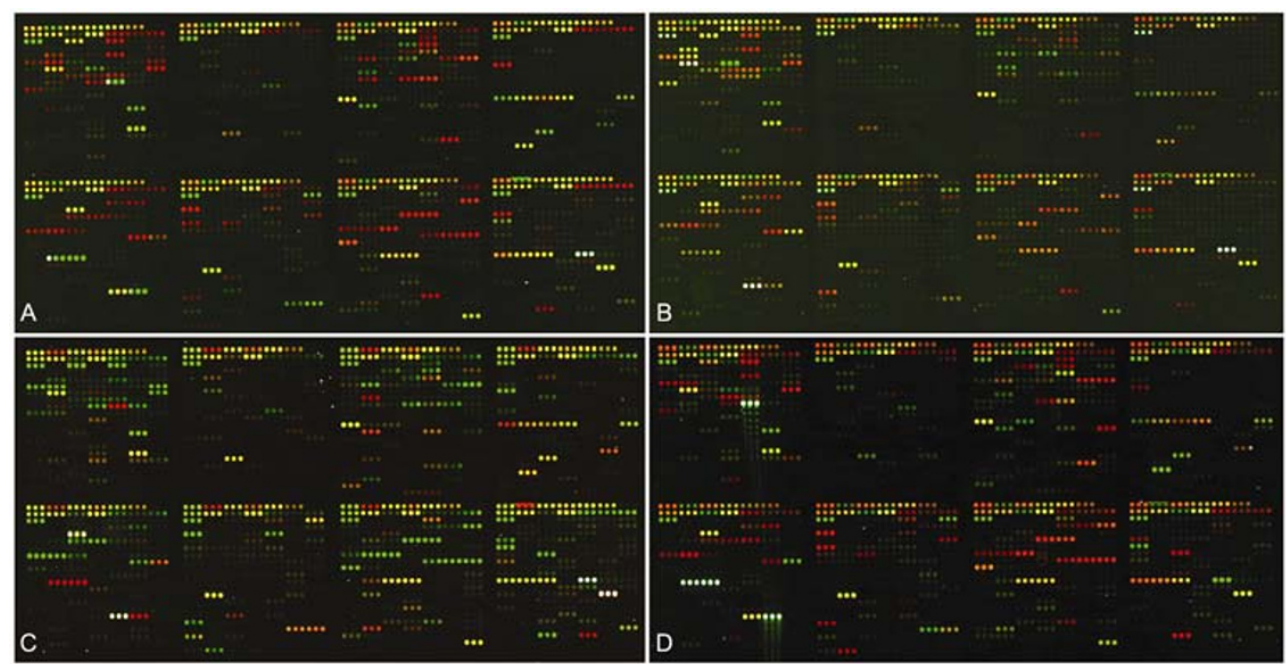

Figure 2 Pseudo-color map for chip hybridization. A, Paired N3+T4; B, N4+T3; C, T1+N2; D, N1+T2. 
Table 2 miRNA chips test results ${ }^{\text {a) }}$

\begin{tabular}{cccc}
\hline Gene ID & Score $(d)$ & Fold change & $Q$-value $(\%)$ \\
\hline hsa-miRNA-20a & 5.013 & 75.827 & 0 \\
hsa-miRNA-106a & 4.733 & 38.875 & 0 \\
hsa-miRNA-34a & 4.476 & 37.806 & 0 \\
hsa-miRNA-21 & 4.203 & 27.260 & 0 \\
hsa-miRNA-17 & 4.282 & 18.797 & 0 \\
hsa-miRNA-145 & -4.766 & 0.040 & 0 \\
hsa-miRNA-204 & -3.629 & 0.024 & 0 \\
\hline
\end{tabular}

a) Table 2 shows miRNA with the maximum factor of the differential expression. Up-regulated miRNA: $75.8 \times$ for miRNA-20a; $38.9 \times$ for miRNA$106 \mathrm{a} ; 37.8 \times$ for miRNA-34a; $27.3 \times$ for miRNA-21; and $18.8 \times$ for miRNA-17. Down-regulated miRNA: $25 \times$ for miRNA-145; $50 \times$ for miRNA-204.

20a in uveal melanoma was 4.5 times higher than in normal human tissues. The expressions of hsa-miRNA-106a and hsa-miRNA-21 were respectively 4.5 and 2 times higher than normal. The expression of hsa-miRNA-17 was 2.8 times higher, while the expression of hsa-miRNA-34a was
6.6 times higher. However, the expressions of hsa-miRNA145 and hsa-miRNA-204 in uveal melanoma were 30.4 and 42.5 times lower than in normal tissues (Figure 4 and Table 3).

\section{Discussion}

Tumor results from uncontrolled cellular proliferation and apoptosis. There are several protective measures to ensure that developing cells and mature cells normally divide, differentiate, and undergo apoptosis. Multiple regulatory factors, through the gene expression switches, define cell mitosis and differentiation. An imbalance between the tumor suppressor gene and the oncogene may cause tumorgenesis. Most tumor suppressor genes and oncogenes are transcribed into RNA from DNA, before they are translated into proteins and conduct their biological functions. A kind of RNA
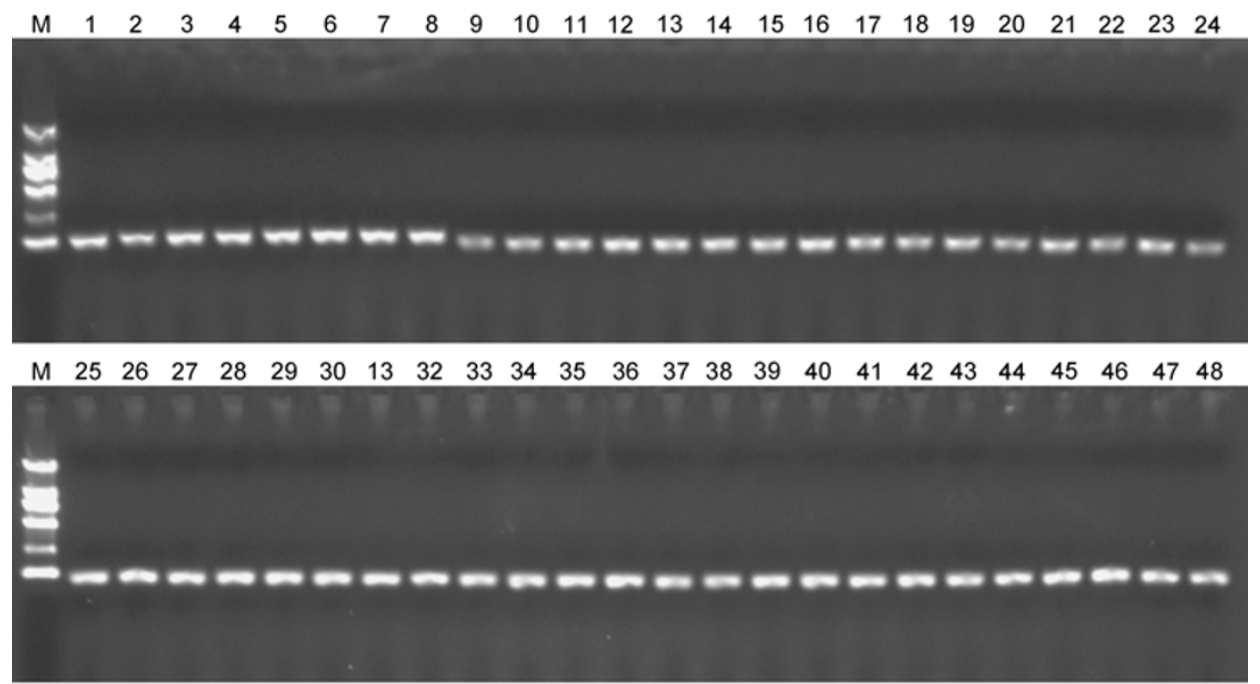

$\begin{array}{lllllllllllllllllllllllll}\text { M } & 49 & 50 & 51 & 52 & 53 & 54 & 55 & 56 & 57 & 58 & 59 & 60 & 61 & 62 & 63 & 64 & 65 & 66 & 67 & 68 & 69 & 70 & 71 & 72\end{array}$
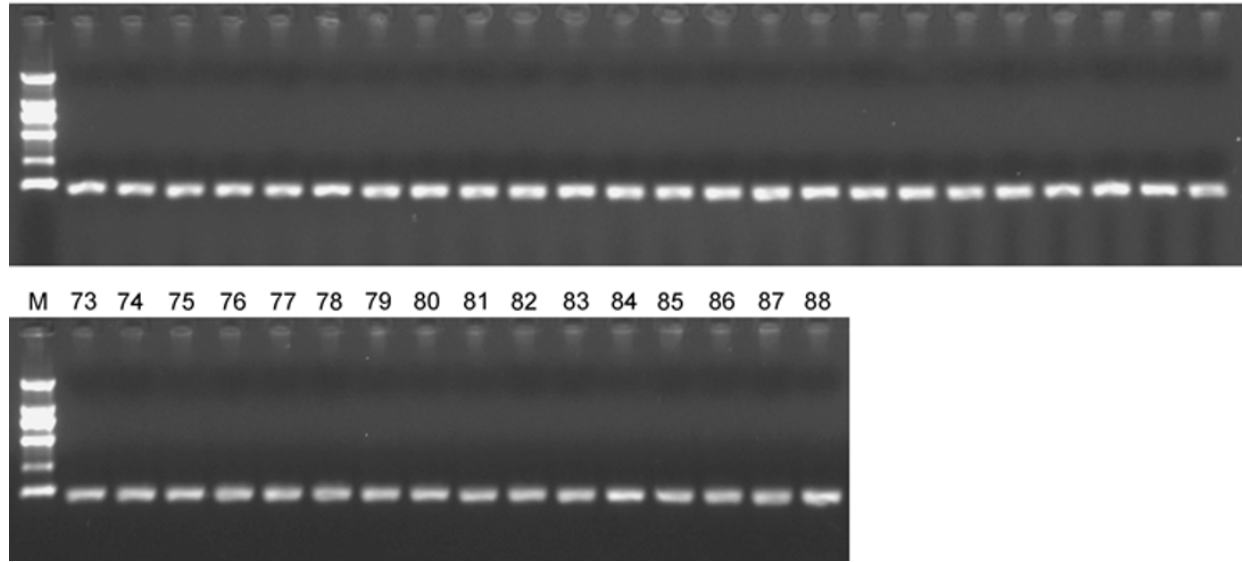

Figure 3 The following channels used 1st-cDNAs of samples of N4, N1, N3, N2, T3, T1, T3 and T4 as templates for each electrophoresis result of miRNA. 1-8, Real time PCR amplified U6 gene; 9-16, Real time PCR amplified hsa-miRNA-20a gene; 17-24, Real time PCR amplified hsa-miRNA-106a gene; 25-32, Real time PCR amplified hsa-miRNA-21 gene; 33-40, Real time PCR amplified hsa-miRNA-17 gene; 41-48, Real time PCR amplified hsa-miRNA-29a gene; 49-56, Real time PCR amplified hsa-miRNA-143 gene; 57-64, Real time PCR amplified hsa-miRNA-145 gene; 65-72, Real time PCR amplified hsa-miRNA-204 gene; 73-80, Real time PCR amplified hsa-miRNA-let-7g gene; 81-88, Real time PCR amplified hsa-miRNA-34a gene. Marker TaKaRa DL2000. The lengths of the bands were in the following order from top to bottom: 100, 250, 500, 750, 1000, and $2000 \mathrm{bp}$. 
Table 3 RT-PCR test results ${ }^{\text {a) }}$

\begin{tabular}{|c|c|c|c|c|c|}
\hline Number & Name & Crossing point & $\mathrm{E}$ & $\Delta \mathrm{CP}($ con-sam $)$ & Ratio after normalization \\
\hline 1 & N4-U6 & 18.75 & & & \\
\hline 2 & N1-U6 & 19.42 & 1.805 & -0.67 & \\
\hline 3 & N3-U6 & 19.58 & & -0.83 & \\
\hline 4 & N2-U6 & 20.16 & & -1.41 & \\
\hline 5 & T3-U6 & 19.99 & & -1.24 & \\
\hline 6 & T1-U6 & 20.07 & & -1.32 & \\
\hline 7 & T2-U6 & 19.44 & & -0.69 & \\
\hline 8 & T4-U6 & 20.41 & & -1.66 & \\
\hline 9 & $\mathrm{~N} 4-20 \mathrm{a}$ & 23.34 & & & \\
\hline 10 & N1-20a & 23.87 & 1.797 & -0.53 & 1.088752706 \\
\hline 11 & N3-20a & 24.2 & & -0.86 & 0.986199402 \\
\hline 12 & $\mathrm{~N} 2-20 \mathrm{a}$ & 24.04 & & -0.7 & 1.525629953 \\
\hline 13 & T3-20a & 22.76 & & 0.58 & 2.921928742 \\
\hline 14 & T1-20a & 21.87 & & 1.47 & 5.161020517 \\
\hline 15 & T2-20a & 20.74 & & 2.6 & 6.899129116 \\
\hline 16 & $\mathrm{~T} 4-20 \mathrm{a}$ & 22.73 & & 0.61 & 3.810890669 \\
\hline 17 & N4-106a & 25.69 & & & \\
\hline 18 & N1-106a & 26.27 & 1.816 & -0.58 & 1.050878577 \\
\hline 19 & N3-106a & 26.65 & & -0.96 & 0.920714263 \\
\hline 20 & N2-106a & 26.85 & & -1.16 & 1.150955365 \\
\hline 21 & T3-106a & 25.2 & & 0.49 & 2.786111542 \\
\hline 22 & T1-106a & 24.53 & & 1.16 & 4.356365314 \\
\hline 23 & T2-106a & 22.99 & & 2.7 & 7.526309119 \\
\hline 24 & T4-106a & 25.23 & & 0.46 & 3.507078207 \\
\hline 25 & N4-21 & 21.45 & & & \\
\hline 26 & N1-21 & 22.12 & 1.81 & -0.67 & 0.998148327 \\
\hline 27 & N3-21 & 22.43 & & -0.98 & 0.912748395 \\
\hline 28 & $\mathrm{~N} 2-21$ & 22.48 & & -1.03 & 1.24802685 \\
\hline 29 & T3-21 & 21.75 & & -0.3 & 1.74071697 \\
\hline 30 & $\mathrm{~T} 1-21$ & 21 & & 0.45 & 2.847772363 \\
\hline 31 & $\mathrm{~T} 2-21$ & 21.15 & & 0.3 & 1.795861519 \\
\hline 32 & $\mathrm{~T} 4-21$ & 21.46 & & -0.01 & 2.649569871 \\
\hline 33 & N4-17 & 23.88 & & & \\
\hline 34 & N1-17 & 24.01 & 1.802 & -0.13 & 1.375915002 \\
\hline 35 & N3-17 & 24.61 & & -0.73 & 1.062123669 \\
\hline 36 & N2-17 & 24.43 & & -0.55 & 1.663289049 \\
\hline 37 & T3-17 & 23.58 & & 0.3 & 2.481750807 \\
\hline 38 & $\mathrm{~T} 1-17$ & 22.93 & & 0.95 & 3.815196926 \\
\hline 39 & $\mathrm{~T} 2-17$ & 21.83 & & 2.05 & 5.026510394 \\
\hline 40 & $\mathrm{~T} 4-17$ & 23.71 & & 0.17 & 2.945984626 \\
\hline 41 & N4-34a & 27.08 & & & \\
\hline 42 & N1-34a & 27.9 & 1.801 & -0.82 & 0.916892634 \\
\hline 43 & N3-34a & 27.86 & & -0.78 & 1.031752156 \\
\hline 44 & $\mathrm{~N} 2-34 \mathrm{a}$ & 28.16 & & -1.08 & 1.218086278 \\
\hline 45 & T3-34a & 24.76 & & 2.32 & 8.143733431 \\
\hline 46 & $\mathrm{~T} 1-34 \mathrm{a}$ & 25.86 & & 1.22 & 4.469682164 \\
\hline 47 & $\mathrm{~T} 2-34 \mathrm{a}$ & 24.85 & & 2.23 & 5.581678937 \\
\hline 48 & $\mathrm{~T} 4-34 \mathrm{a}$ & 24.93 & & 2.15 & 9.442927274 \\
\hline 49 & N4-145 & 19.38 & & & \\
\hline 50 & N1-145 & 21.28 & 1.803 & -1.9 & 0.484671907 \\
\hline 51 & N3-145 & 20.55 & & -1.17 & 0.819145559 \\
\hline
\end{tabular}




\begin{tabular}{|c|c|c|c|c|c|}
\hline Number & Name & Crossing point & $\mathrm{E}$ & $\Delta \mathrm{CP}($ con-sam $)$ & Ratio after normalization \\
\hline 52 & $\mathrm{~N} 2-145$ & 20.53 & & -1.15 & 1.167448617 \\
\hline 53 & T3-145 & 27.08 & & -7.7 & 0.02222617 \\
\hline 54 & $\mathrm{~T} 1-145$ & 27.55 & & -8.17 & 0.017663014 \\
\hline 55 & $\mathrm{~T} 2-145$ & 26.45 & & -7.07 & 0.023285174 \\
\hline 56 & $\mathrm{~T} 4-145$ & 26.07 & & -6.69 & 0.051658376 \\
\hline 57 & N4-204 & 16.31 & & & \\
\hline 58 & N1-204 & 18.69 & 1.811 & -2.38 & 0.361404717 \\
\hline 59 & N3-204 & 17.94 & & -1.63 & 0.620110389 \\
\hline 60 & N2-204 & 17.94 & & -1.63 & 0.873425338 \\
\hline 61 & T3-204 & 24.88 & & -8.57 & 0.012813584 \\
\hline 62 & T1-204 & 25.97 & & -9.66 & 0.007031657 \\
\hline 64 & T4-204 & 23.17 & & -6.86 & 0.045334788 \\
\hline
\end{tabular}

a) Verification demonstrated that the RT-PCR results matched the chip results. N4 was the control; N1, N3, N2, T3, T1, T3, and T4 were the samples; ratio $=$ sample/control.

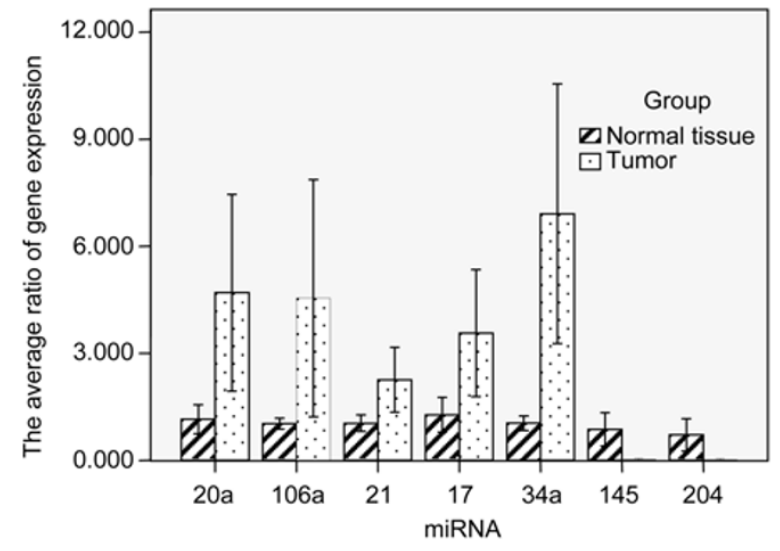

Figure 4 The results of miRNA RT-PCR in normal human choroidal tissue and uveal melanoma. We used the $t$-test for the statistical analysis of mRNA expression in normal human choroidal tissue and uveal melanoma. We can see that miRNA-20a, 106a, 17, 21 and 34a were up-regulated, and miRNA-145 and miRNA-204 down-regulated.

molecule of non-encoding protein, also known as microRNAs (miRNAs), and the mutation or ectopic expression of miRNAs have been conceptionally related with many human tumors, as miRNAs may act as a tumor suppressor gene or as an oncogene. It has been shown that the inhibited expression of major tumor-related genes assists in diagnosis and treatment. As a highly effective regulatory factor for gene expression, miRNA facilitates diagnosis and treatment in uveal melanoma. Lori et al. [4] used a miRNA microarray to detect the expression level in uveal melanoma with metastasis. They discovered that let-7b and miRNA-199a had a high sensitivity and specificity for differentiating metastatic melanoma. In comparing uveal or benign neoplasms with tumor tissues, it is possible to detect specifically upregulated or down-regulated miRNA, as well as the variations among different types of uveal melanoma. For example, high expression of specific miRNA signified its effect as an oncogene, while low expression or non-expression suggested an effect as a tumor suppressor gene.

As a fast and high quantity test, microRNA chips (CapitalBio Company) were adopted to initially establish the miRNAs expression profile in uveal melanoma. The up-regulated genes in uveal melanoma included hsa-miRNA-20a, hsa-miRNA-106a, hsa-miRNA-34a, hsa-miRNA-17, and hsa-miRNA-21. The down-regulated genes included hsa-miRNA-145 and hsa-miRNA-204, of which the expressions were much lower than those in normal tissues. In this study, we aimed at largely differently expressed miRNAs between uveal melanoma and normal human uveal tissues.

He et al. [5] described the relationship among miRNAs, Myc, and cancer. In scattered B-cell lymphoma, follicular lymphoma, and mantle cell lymphoma, the point $13 \mathrm{q} 31$ gene was frequently amplified, and the gene at this amplified site contained a non-encoding protein RNA, C13orf 25 . This transcription set encoded a gene cluster of miRNA-1792. They discovered that in $65 \%$ of B-cell lymphoma, the expression of precursor miRNA-17-92 was enhanced. They speculated that the hyperactive expression of this gene cluster was related to tumor formation. However, Guil et al. [6] found that miRNA-17-92 was part of expressed intrinsic RNAs, which included miRNA-17, miRNA-18a, miRNA19a, miRNA-20a, miRNA-19b-1 and miRNA-92. These miRNAs were believed to have potential to be oncogenes.

Tanzer et al. [7] pointed out that miRNA-106a and miRNA-17 were homogenous in origin. Therefore, miRNA106a was an oncogene. In the cases of human solid tumors, miRNA-106a was expressed in colon cancer, pancreatic cancer, and prostate cancer, while miRNA-92-2 was expressed in pancreatic cancer, prostate cancer, and gastric carcinoma. In Xiao's [8] research, the level of miRNA-106a in tumor tissues was much higher than in non-tumor tissues, 
and this was clearly related to tumor staging, size, differentiation, lymphatic or remote metastasis, and infiltration.

Many reports noted that miRNA-21 was abnormally expressed in many tumors. Volinia et al. [9] conducted a miRNA chip screening for 540 cases of human samples, in which 363 cases came from six types of solid tumors: pulmonary carcinoma, breast cancer, gastric carcinoma, prostate cancer, colon cancer, and pancreatic cancer. Research found that miRNA-21 was closely related to the development and survival of many tumors. An abnormally high expression of miRNA-21 was a common phenomenon in many different tumors. A more elevated abnormal expression of miRNA-21 in chronic lymphatic leukemia, hepatic tumor, tumors of the neck and face, and human osteosarcoma was found. Kunz et al. [10] found that in patients of metastatic melanoma, in comparison to the non-treatment control group and under different treatment approaches, the expression of miRNA-21 was down-regulated after treatment. This study was the first to report where miRNA responded to chemotherapy and was a key factor in the pathogenic mechanism of melanoma. In gene therapy for uveal melanoma this may provide a pathway.

O'Donnell et al. [11] pointed out that miRNA-20a, with the effect of a tumor suppressor gene, targets E2F1, thereby reducing the effect of E2F1. Comparison was made between tumors with only Myc over-expressed and lymphoma with both Myc and the miRNA-17-19-b1 gene cluster superexpressed. The latter had a stronger proliferation ability and a lower cell death rate. E2F1 regulated Myc through a positive feedback loop. O'Donnell et al. also found that with existing Myc, miRNAs in the miRNA-17-92 gene cluster restrained the activity of E2F1. By blocking Myc and the positive feedback loop of E2F1, the influence of Myc on cell proliferation was diminished. In this model, the effect of the miRNA-17-92 gene cluster was the effect of tumor suppressor gene, which was the opposite of the discovery of He et al. [5]. Consistent with this model, the point 13q31 gene encoding miRNA-17-92 in hepatic carcinoma was missing. Although E2F1 promoted cell proliferation, it also induced apoptosis when its expression level surpassed the threshold. In this situation, the negative control on E2F1 by miRNAs was achieved through blocking the inductive activity of E2F1 for apoptosis, simultaneously enhancing Myc-mediated cell proliferation. This idea supported the model reported by $\mathrm{He}$ [5]. Multiple characteristics of being a tumor suppressor gene and oncogene, as exhibited by miRNA-17-92, suggested the complexity in tumor development and gene regulation by miRNAs.

In research by Yan et al. [12], in the uveal melanoma, miRNA-34a down-regulated C-MET. Through the HGF-dependent Akt signal transduction pathway, it inhibited cell proliferation and migration. However, miRNA-34a, in colon cancer, pancreatic cancer, and neural tumors, also inhibited cell proliferation. This was inconsistent with our research results. This was possibly because they chose a cell strain of uveal melanoma to test miRNA, instead of directly using the human uveal melanoma and normal uveal tissues as we did in our experiment. Zanatte et al. [13], when constructing the expression profile of miRNA for CLL, saw an up-regulation in miRNA-34a. We therefore speculated that in different types of tumor, the regulation method on the target gene by miRNA might vary, where different signal pathways or different checkpoints of a signal pathway might be involved. A single miRNA might have different regulatory approaches for genes of different tumors which remains incompletely understood.

Michael et al. [14] discovered that miRNA-143 and miRNA-145 were sparsely expressed in colorectal cancer. Research suggested that in colorectal cancer, there was a significant drop in the level of mature miRNA-145, although there was not a significant variation in the number of pre-transcribed hairclip structure of miRNAs in both the normal and tumor tissues. It was suggested that there was a maturation problem in miRNA-145 during tumor formation. miRNA-204 has been relatively uninvestigated. Deo et al. [15], in the mammalian choroid plexus, found expression of miRNA-204, while Wu et al. [16] found down-regulated expression of miRNA-204 in human endometrioid adenocarcinoma.

Our experiment showed that hsa-miRNA-20a, hsamiRNA-106a, hsa-miRNA-34a, hsa-miRNA-17, and hsamiRNA-21 were up-regulated, and hsa-miRNA-145, hsamiRNA-204 were down-regulated. In most tumor research, hsa-miRNA-20a, 106a, and 17 were specifically up-regulated, while hsa-miRNA-145 and 204 were down-regulated. These miRNAs were factors inducing abnormal proliferation in tumors, suggesting that uveal melanoma originated from these abnormally proliferated tissues. miRNA- 21 was strongly correlated to the metastasis of uveal melanoma. In this experiment, there were three cases of spindle cell type uveal melanoma with a low probability of metastasis and one case of mixed type uveal melanoma with primarily spindle cell type. Their expressions of miRNA-21 were low, which were in accordance to Bandrés's results [17]. As the pathological grade of a tumor increased (suggesting more malignancy), the expression of miRNA-21 also increased. The question of inhibiting expression of miRNA-21 to reduce the metastatic property of uveal melanoma requires further investigation. From research results about the miRNA of various tumors, it has been demonstrated that miRNA had a significant function in inhibiting gene activities in tumors and was possibly influential in developing gene therapy for tumors. Similar to the method of comparing miRNA levels in tumor and normal tissues, scanning for a large area of miRNA expression identifies more tumor-related miRNA. Induction of anti-sense oligodeoxynucleotides with combined miRNA, which possesses the characteristics of an oncogene, also known as anti-miRNA oligodeoxynucleotides (AMOs), may deactivate miRNAs in tumors, elongate patient survival, and induce tumor death. 
Also, the injection of cholesterol-linked AMOs in rats effectively inhibits the activity of miRNA in different organs, and thus, may treat disease. The use of viruses or liposomes as an expression system immediately induces a large quantity of miRNAs. These techniques ensure, under control by a specific promoter of certain tissues, that pre-miRNA is expressed, as well as the sequences on both sides, while the refinement of intrinsic miRNA is stimulated to produce correct miRNA for inhibiting specific gene expression. However, these methods were usually limited by immunological responses, which prevented effective transport of RNA. The transition of miRNA therapy from laboratory conditions to clinical application will require further refinement.

\section{Conclusion}

In this experiment, we had initially obtained a result indicating differential expressions of miRNA in uveal melanoma. Unfortunately, uveal melanoma tissues and normal human uveal tissues are difficult to obtain. This study did not involve a classification study of uveal melanoma, but rather was a preliminary study about cases of uveal melanoma, which were collected from local surgical resection. In the future, with increasing case numbers and classifying of uveal melanoma, we plan to conduct a deeper study to understand its pathogenic mechanism to seek possible modes for gene therapy, which may provide a more convenient and effective way for clinical therapy.

This work was supported by the Beijing Municipal Natural Science Foundation (Grant Nos. 7092021 and 7072019), the Beijing Science and Technology Support (Grant No. Z08050703080882) and the National Natural Science Foundation of China (Grant No. 30571988).
1 Bedikian A Y. Metastatic uveal melanoma therapy: current options. Int Ophthalmol Clin, 2006, 46: 151-166

2 He L, Thomson J M, Hemann M T, et al. A microRNA polycistron as a potential human oncogene. Nature, 2005, 435: 823-833

3 Zamore P D, Haley B. Ribo-gnome: the big world of small RNAs. Science, 2005, 309: 1519-1524

4 Lori AW, Meghan D L, Michael D O, et al. Micro-RNAs associated with metastasis in uveal melanoma identified by multiplexed microarray profiling. Melanoma Res, 2008, 18: 184-190

5 He L, Thomson J M, Hemann M T, et al. A microRNA polycistron as a potential human oncogene. Nature, 2005, 435: 828-833

6 Guil S, Cáceres J F. The multifunctional RNA-binding protein hnRNP A1 is required for processing of miRNA-18a. Nat Struct Mol Biol, 2007, 14: 591-596

7 Tanzer A, Stadler P F. Molecular evolution of a microRNA cluster. J Mol Biol, 2004, 339: 327-335

8 Xiao B, Guo J, Miao Y, et al. Detection of miRNA-106a in gastric carcinoma and its clinical significance. Clin Chim Acta, 2009, 400: 97-102

9 Volinia S, Calin G A, Liu C G, et al. A microRNA expression signature of human solid tumors defines cancer gene targets. Proc Natl Acad Sci USA, 2006, 103: 2257-2261

10 Kunz M, Schultz J, Ibrahim S. Role of microRNA in melanoma progression. Melanoma Res, 2006, 16: S53-S54

11 O'Donnell K A, Wentzel E A, Zeller K I, et al. c-Myc-regulated microRNAs modulate E2F1 expression. Nature, 2005, 435: 839-843

12 Yan D, Zhou X, Chen X, et al. MicroRNA-34a inhibits uveal melanoma cell proliferation and migration through downregulation of c-Met. Invest Ophthalmol Vis Sci, 2008, doi: 10.1167/iovs.08-2681

13 Zanette D L, Rivadavia F, Molfetta G A, et al. miRNA expression profiles in chronic lymphocytic and acute lymphocytic leukemia. Braz J Med Biol Res, 2007, 40: 1435-1440

14 Michael M Z, O'Connor S M, van Holst Pellekaan N G, et al. Reduced accumulation of specific microRNAs in colorectal neoplasia. Mol Cancer Res, 2003, 1: 882-891

15 Deo M, Yu J Y, Chung K H, et al. Detection of mammalian microRNA expression by in situ hybridization with RNA oligonucleotides. Dev Dyn, 2006, 235: 2538-2548

$16 \mathrm{Wu} \mathrm{W}$, Lin Z, Zhuang Z, et al. Expression profile of mammalian microRNAs in endometrioid adenocarcinoma. Eur J Cancer Prev, 2009, 18: 50-55

17 Bandrés E, Cubedo E, Agirre X, et al. Identification by Real-time PCR of 13 mature microRNAs differentially expressed in colorectal cancer and non-tumoral tissues. Mol Cancer, 2006, 5: 29

Open Access This article is distributed under the terms of the Creative Commons Attribution License which permits any use, distribution, and reproduction in any medium, provided the original author(s) and source are credited. 